\title{
Combination of Evidential Sensor Reports with Distance Function and Belief Entropy in Fault Diagnosis
}

\author{
Y. Dong, J. Zhang, Z. Li, Y. Hu, Y. Deng
}

\section{Yukun Dong}

Institute of Fundamental and Frontier Science

University of Electronic Science and Technology of China, Chengdu, 610054, China

\author{
Jiantao Zhang \\ College of Information Science and Technology \\ Jinan University, Tianhe, Guangzhou, 510632, China \\ Zhen Li \\ College of Information Science and Technology \\ Jinan University, Tianhe, Guangzhou, 510632, China

\section{Yong $\mathrm{Hu}$} \\ Big Data Decision Institute \\ Jinan University, Tianhe, Guangzhou, 510632, China

\section{Yong Deng*} \\ 1. Institute of Fundamental and Frontier Science \\ University of Electronic Science and Technology of China, Chengdu, 610054, China \\ 2. Big Data Decision Institute \\ Jinan University, Tianhe, Guangzhou, 510632, China \\ *Corresponding author: dengentropy@uestc.edu.cn; prof.deng@hotmail.com
}

\begin{abstract}
Although evidence theory has been applied in sensor data fusion, it will have unreasonable results when handling highly conflicting sensor reports. To address the issue, an improved fusing method with evidence distance and belief entropy is proposed. Generally, the goal is to obtain the appropriate weights assigning to different reports. Specifically, the distribution difference between two sensor reports is measured by belief entropy. The diversity degree is presented by the combination of evidence distance and the distribution difference. Then, the weight of each sensor report is determined based on the proposed diversity degree. Finally, we can use Dempster combination rule to make the decision. A real application in fault diagnosis and an example show the efficiency of the proposed method. Compared with the existing methods, the method not only has a better performance of convergence, but also less uncertainty.

Keywords: Dempster-Shafer evidence theory, sensor data fusion, fault diagnosis, evidence distance, belief entropy, information volume.
\end{abstract}

\section{Introduction}

In mechanical engineering, some systems are very complex, which might have many components, reflecting with each other $[11,38,44,71]$. It is likely that something happens unexpectedly in the systems and causes serious problems due to a variety of reasons, such as unfavorable weather, bad environment or a long time of working. As a result, making full use of sensor reports information is extremely significant to make a reasonable decision in fault diagnosis $[58,81]$.

In order to make a rational decision when using sensor data fusion technology, some works have been proposed to handle uncertainty [39, 45, 59,77], such as fuzzy set theory $[15,63,66$, $74,76,83], \mathrm{Z}$ numbers [30,31], D numbers [8,9,41,64,65], $\mathrm{R}$ numbers [47,48] and so on. One 
of the most used math tools in sensor data fusion is evidence theory [4,49]. This theory can efficiently model uncertain information with basic probability assignment (BPA), or called as belief function [21]. In addition, the Dempster rule can efficiently combine the sensor reports from different sources [79]. Due to the desirable properties, evidence theory has been accepted as de facto standard in decision making $[10,28,78]$, risk and reliability analysis $[13,27,40]$, system optimization [67] and pattern recognition [23, 24,34,46,57].

However, an open issue of evidential sensor data fusion is that the illogical results will be obtained when sensor reports conflict with each other in a high degree [20,56,75]. Many methods were presented to address this issue [60,80]. For example, Yager [70] removed the process of normalizing in D-S combination rule, Smets [50,51] proposed the conjunctive and disjunctive rules, Murphy [42] combined the conflicting evidence with average operation, Fan and Zuo [16] presented a combination method to fuse conflicting evidence in fault diagnosis, Dubois and Prades method [12], Lefevre et al. [33] and so on. Recently, Jiang et al. [25] applied belief entropy into sensor data fusion and received the best performance.

Although these methods have some advantages, one of the disadvantages is that some information is not fully used. For example, the distance information and the difference of information volume are not considered in [25]. In this paper, we proposed an improved evidential method, which is conceptually simple, and yet is able to provide much better accuracy and less uncertainty. The basic idea is to obtain the appropriate weights for different reports. The distribution difference between two BPAs is first measured by belief entropy [6]. Then the diversity degree among BPAs can be obtained by combining distribution difference and evidence distance [29]. According to it, the weight of each BPA can be determined. Finally, we can make a decision for fault diagnosis by using Dempster combination rule. An application in fault diagnosis and an example show that our proposed approach can not only increase the accuracy of fault diagnosis but also decrease the uncertain information volume, which is more reasonable.

The remainder of this paper is organized as follows. Section 2 introduces some backgrounds, including Dempster-Shafer Evidence Theory, Evidence Distance and Belief entropy. Section 3 formulates the proposed method for evidential sensor data fusion. A real application in fault diagnosis and an example are given in Section 4 to show the efficiency of the method. The conclusions are in Section 5 .

\section{Preliminaries}

\subsection{Dempster-Shafer Evidence Theory}

The application in data fusion needs efficient math tools [38]. Dempster-Shafer Evidence Theory, proposed by Dempster [4] and Shafer [49], is effective to handle uncertain information.

Definition 1. Let $X$ be a set of mutually exclusive and collectively exhaustive events, shown as follow $[4,49]$ :

$$
X=\left\{\theta_{1}, \theta_{2}, \cdots, \theta_{i}, \cdots, \theta_{|X|}\right\}
$$

where set $X$ is called a frame of discernment, whose power set is:

$$
2^{X}=\left\{\emptyset,\left\{\theta_{1}\right\}, \cdots,\left\{\theta_{|X|}\right\},\left\{\theta_{1}, \theta_{2}\right\}, \cdots,\left\{\theta_{1}, \theta_{2}, \cdots, \theta_{i}\right\}, \cdots, X\right\}
$$

Definition 2. For a frame of discernment $X=\left\{\theta_{1}, \theta_{2}, \cdots, \theta_{|X|}\right\}$, a mass function is a mapping $m$ from $2^{\theta}$ to $[0,1]$.

$$
m: 2^{\theta} \rightarrow[0,1]
$$


which satisfies the following condition:

$$
m(\emptyset)=0, \sum_{A \in 2^{X}} m(A)=1
$$

$A$, a member of the power set, is called a focal element of the mass function, or named as basic probability assignment (BPA).

BPA is the key issue in evidence theory and many relative processing are presented such as negation [19,73], correlation [26] and divergence measure [17,52].

Definition 3. Given two BPAs, $m_{1}$ and $m_{2}$, they can be combined by,

$$
m(A)= \begin{cases}0, & A=\emptyset \\ \frac{1}{1-K} \sum_{B \cap C=A} m_{1}(B) m_{2}(C), & A \neq \emptyset\end{cases}
$$

with

$$
K=\sum_{B \cap C=\emptyset} m_{1}(B) m_{2}(C)
$$

where $K$ is a parameter that reflects the conflict between $m_{1}$ and $m_{2}$. If $K=0, m_{1}$ and $m_{2}$ have no contradiction.

If $K=1$, they are totally conflict. Many open issues about conflict management are still not well addressed [2]. Some alternatives are proposed to modify the combination rule $[53,54,61,68]$, others are presented to modify the data models [80] or handle this problem under open world assumption $[55,56]$.

\subsection{Evidence distance}

Definition 4. Let $m_{1}$ and $m_{2}$ be two BPAs on the same frame of discernment of $X$, which contains $N$ mutually exclusive and exhaustive hypotheses. The distance between $m_{1}$ and $m_{2}$ is [29]:

$$
d_{B P A}\left(m_{1}, m_{2}\right)=\sqrt{\frac{1}{2}\left(\overrightarrow{m_{1}}-\overrightarrow{m_{2}}\right)^{T} D\left(\overrightarrow{m_{1}}-\overrightarrow{m_{2}}\right)}
$$

where $\mathrm{D}$ is a $2^{N} \times 2^{N}$ matrix whose elements are

$$
D(A, B)=\frac{|A \cap B|}{|A \cup B|}, \quad A, B \in P(X) .
$$

\subsection{Belief entropy}

It should be pointed out that uncertainty measurement, decision making and optimization under uncertainty is still an open issue $[14,22,37]$. Entropy is an efficient tool to model uncertainty $[7,32,72]$. Recently, a new belief entropy, named as Deng entropy was proposed [6]. It has a good performance in measuring uncertainty. Also, it has a backward compatibility, which means when the uncertain information is represented by probability distribution, belief entropy will degenerate to Shannon entropy $[1,3,35,43]$.

Definition 5. Let $A$ be a proposition of BPA, $|A|$ is the cardinality of $A$. Then, belief entropy is defined as [6]:

$$
E_{d}=-\sum_{A \subseteq X} m(A) \log \frac{m(A)}{2^{|A|}-1}
$$


When the BPA has only one element, which means $|A|=1$, then it can be written as Shannon entropy [6].

$$
E_{d}=-\sum_{A \subseteq X} m(A) \log \frac{m(A)}{2^{|A|}-1}=-\sum_{A \subseteq X} m(A) \log m(A)
$$

\section{Evidential sensor data fusion}

This section formulates a new weighted average approach for evidential sensor data fusion. The proposed method considered both evidence distance and belief entropy to obtain the appropriate weights assigning to different data reports. Using the weight to pre-treat the multi-source reports, and making the final decision by Dempster combination rule. The flow chart is shown in Figure 1.

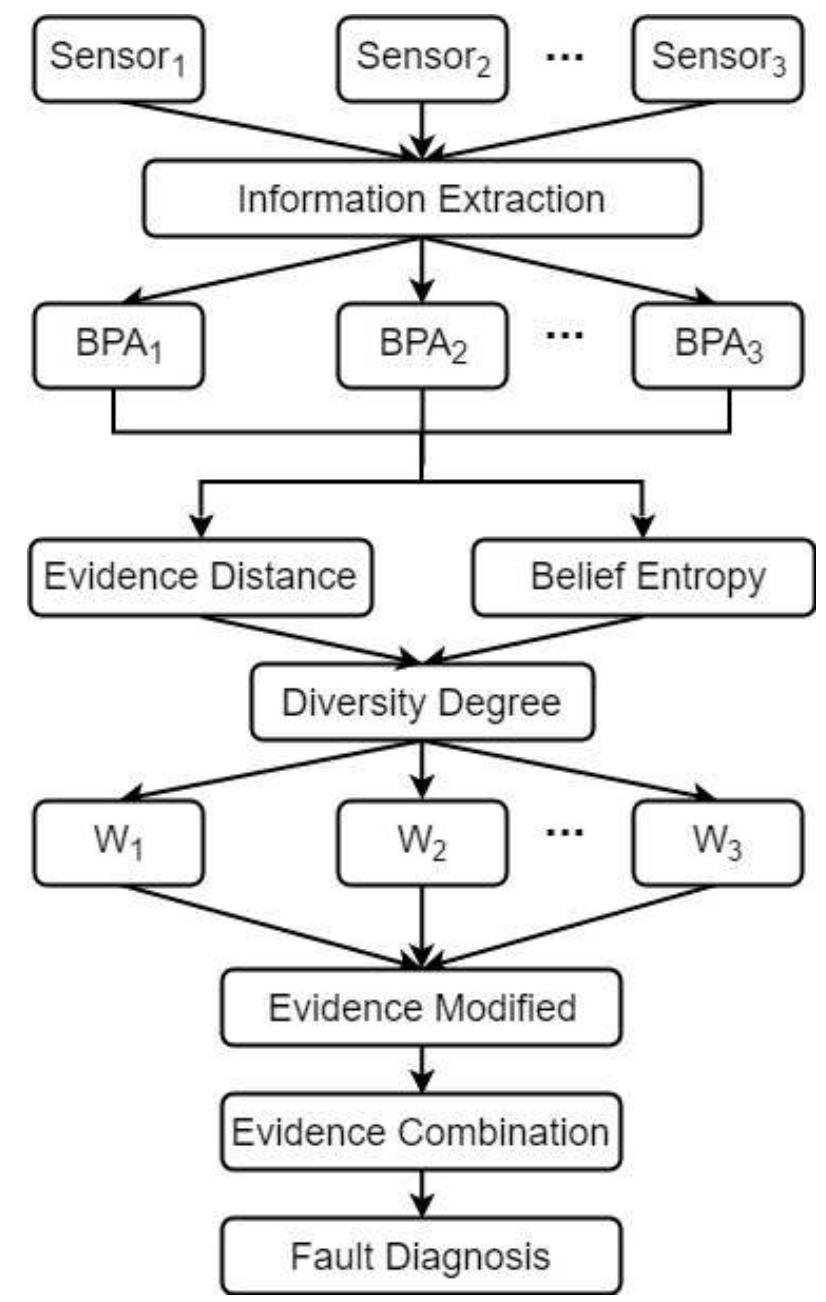

Figure 1: Structure of the proposed sensor data fusing method

Definition 6. Let $E_{d i}, E_{d j}$ be the belief entropy of $m_{1}, m_{2}$, then the distribution difference is defined:

$$
\alpha_{i j}=e^{\left|E_{d i}-E_{d j}\right|}
$$

Definition 7. Let $d_{B P A}\left(m_{i}, m_{j}\right)$ be the evidence distance of $m_{1}, m_{2}$, the diversity degree between 
two BPAs is defined:

$$
D_{i j}=\frac{1}{\alpha_{i j} \times d_{B P A}\left(m_{i}, m_{j}\right)}
$$

Definition 8. A diversity of distribution and distance matrix $(D D D)$ is also defined:

$$
D D D=\left(\begin{array}{cccccc}
1 & D_{12} & \cdots & D_{1 i} & \cdots & D_{1 n} \\
D_{21} & 1 & \cdots & D_{2 i} & \cdots & D_{2 n} \\
\vdots & \vdots & \ddots & \vdots & \ddots & \vdots \\
D_{i 1} & D_{i 2} & \cdots & 1 & \cdots & D_{i n} \\
\vdots & \vdots & \ddots & \vdots & \ddots & \vdots \\
D_{n 1} & D_{n 2} & \cdots & D_{n i} & \cdots & 1
\end{array}\right)
$$

The computation step is shown as following.

- Step 1, collect sensor information and transform into BPAs.

- Step 2, use Equation (7) to calculate the evidence distance between two BPAs, which shows the difference.

- Step 3, use Equation (8) to calculate the belief entropy of BPA, which shows the distributive characteristic.

- Step 4, calculate the diversity degree between two BPAs.

- Step 5, the support of the BPAs is given as:

$$
\operatorname{Sup}\left(m_{i}\right)=\sum_{j=1, j \neq i}^{n} D_{i j}
$$

- Step 6, the credibility degree of the BPAs is obtained.

$$
\operatorname{Crd}_{i}=\frac{\operatorname{Sup}\left(m_{i}\right)}{\sum_{i=1}^{n} \operatorname{Sup}\left(m_{i}\right)}
$$

- Step 7, it is easy to see that $\sum_{i=1}^{n} C r d_{i}=1$. As a result, $C r d_{i}$ can be the weight of each BPA. $\left(\omega_{1}, \omega_{2}, \cdots, \omega_{n}\right)=\left(C r d_{1}, C r d_{2}, \cdots, C r d_{n}\right)$. Thus, a new weighted evidence can be obtained, which is:

$$
m(a)=\omega_{1} \times m_{1}(a)+\omega_{2} \times m_{2}(a)+\cdots+\omega_{n} \times m_{n}(a)
$$

- Step 8, use Dempster combination rule to combine the new weighted evidence to get the result. Furthermore, if the number of original evidence is $n$, then the new evidence should be combined for $(n-1)$ times [25].

It should be noticed that if $d_{B P A}\left(m_{i}, m_{j}\right)=0$, which means that there is no conflict between $m_{i}$ and $m_{j}$. In this situation, the value of diversity degree $D_{i j}$ cannot be determined. So we proposed our own solution, which can be discussed further.

First, if there are only three BPAs $m_{1}, m_{2}, m_{3}$ and $m_{1}=m_{2}$, the two BPAs can be regarded as the same one, then use Murphy's method, which is assigning the weight equally to each BPA. 
Second, if there are more than three evidences. Supposing there are $n$ BPAs, which can be divided into $m$ groups according to their $d_{B P A}$. If there are only two groups, it will just be the same as the first situation. If there are more than two groups, and each group has $k$ BPAs, noted as $k_{1}, k_{2}, \cdots, k_{m}$, and $\sum_{i=1}^{m} k_{i}=n$.

$$
\left.\begin{array}{cccc}
\left\{m_{11}\right. & m_{12} & \cdots & \left.m_{1 k_{1}}\right\} \\
\left\{m_{21}\right. & m_{22} & \cdots & \left.m_{2 k_{2}}\right\} \\
\vdots & \vdots & \vdots & \vdots \\
\left\{m_{m 1}\right. & m_{12} & \cdots & m_{m k_{m}}
\end{array}\right\}
$$

Then, select one BPA from each group. Suppose they are $m_{11}, m_{21}, \cdots, m_{m 1}$, and use the proposed method to obtain the weight, which is

$$
\left\{w\left(m_{11}\right), w\left(m_{21}\right), \cdots, w\left(m_{m 1}\right)\right\}=\left\{\omega_{1}, \omega_{2}, \cdots, \omega_{m}\right\}
$$

Since $w=\sum_{i=1}^{m} k_{i} \omega_{i}$, and the finally weight can be gained.

$$
\begin{aligned}
& \left\{\omega_{11}, \omega_{12}, \cdots, \omega_{1 k_{1}}, \omega_{21}, \omega_{22}, \cdots, \omega_{2 k_{2}}, \cdots, \omega_{m 1}, \omega_{m 2}, \cdots, \omega_{m k_{m}}\right\} \\
& =\{\underbrace{\frac{\omega_{1}}{w}, \frac{\omega_{1}}{w}, \cdots, \frac{\omega_{1}}{w}}_{k_{1}}, \underbrace{\frac{\omega_{2}}{w}, \frac{\omega_{2}}{w}, \cdots, \frac{\omega_{2}}{w}}_{k_{2}}, \cdots, \underbrace{\frac{\omega_{m}}{w}, \frac{\omega_{m}}{w}, \cdots, \frac{\omega_{m}}{w}}_{k_{m}}\}
\end{aligned}
$$

Two examples are given to illustrate how it works.

Example 1. Suppose the frame of discernment is $X=\left\{a_{1}, a_{2}, a_{3}\right\}$, and there are three BPAs.

$$
\begin{aligned}
& m_{1}\left(a_{1}\right)=0.3, m_{1}\left(a_{2}, a_{3}\right)=0.7 \\
& m_{2}\left(a_{1}\right)=0.3, m_{2}\left(a_{2}, a_{3}\right)=0.7 \\
& m_{3}\left(a_{1}\right)=0.8, m_{3}\left(a_{2}, a_{3}\right)=0.2
\end{aligned}
$$

Then the weight should be obtained as $W(1 / 4,1 / 4,1 / 2)$.

Example 2. Suppose the frame of discernment is also $X=\left\{a_{1}, a_{2}, a_{3}\right\}$, and there are four BPAs.

$$
\begin{aligned}
& m_{1}\left(a_{1}\right)=0.3, m_{1}\left(a_{2}, a_{3}\right)=0.7, \\
& m_{2}\left(a_{1}\right)=0.3, m_{2}\left(a_{2}, a_{3}\right)=0.7, \\
& m_{3}\left(a_{1}\right)=0.8, m_{3}\left(a_{2}, a_{3}\right)=0.2 . \\
& m_{4}\left(a_{1}\right)=0.6, m_{4}\left(a_{2}, a_{3}\right)=0.4 .
\end{aligned}
$$

In this example, we can just consider $m_{2}, m_{3}$ and $m_{4}$, then use the proposed method to gain the weights, which are

$$
\omega_{2}=0.2585, \omega_{3}=0.3072, \omega_{4}=0.4343
$$

Finally, re-assign the weights to get the result: $W=(0.2054,0.2054,0.2441,0.3451)$. 


\section{Experiments}

\subsection{Application in fault diagnosis}

The complex systems is very complicated since each factor in the system interacting with each other in a very complicated way. To address this issue, network analysis $[18,36,62,69,82]$ and data fusion based technology are presented to deal with complexity and guarantee the reliability of the complex system. [25] gave a case of motor rotor fault diagnosis, where the vibration signal is collected by acceleration sensor $\left(m_{1}\right)$, velocity sensor $\left(m_{2}\right)$, and displacement sensor $\left(m_{3}\right)$. The possible faults including normal operation $\left(F_{1}\right)$, unbalance $\left(F_{2}\right)$, misalignment $\left(F_{3}\right)$, pedestal looseness $\left(F_{4}\right)$. The collected data report is shown in Table 1 , where $X$ is a frame of discernment, and $X=\left\{F_{1}, F_{2}, F_{3}, F_{4}\right\}$.

Table 1: Output of the multi-senors [25]

\begin{tabular}{lllllr}
\hline$m_{i}$ & $F_{1}$ & $F_{2}$ & $F_{3}$ & $F_{4}$ & $\mathrm{X}$ \\
\hline$m_{1}$ & 0.06 & 0.68 & 0.02 & 0.04 & 0.20 \\
$m_{2}$ & 0.02 & 0 & 0.79 & 0.05 & 0.14 \\
$m_{3}$ & 0.02 & 0.58 & 0.16 & 0.04 & 0.20 \\
\hline
\end{tabular}

The computation steps are as following.

By Equation (7), the evidence distance between two BPAs,

$$
d_{B P A}\left(m_{1}, m_{2}\right)=0.7276, \quad d_{B P A}\left(m_{1}, m_{3}\right)=0.1249, \quad d_{B P A}\left(m_{2}, m_{3}\right)=0.6063
$$

Using Equation (8) to get the belief entropy of each BPA.

$$
E_{d 1}=2.1663, \quad E_{d 2}=1.5417, \quad E_{d 3}=2.4232
$$

The distribution difference is obtained by Equation (10).

$$
\alpha_{12}=1.8674, \quad \alpha_{13}=1.2930, \quad \alpha_{23}=2.4145
$$

And the diversity degree calculated by Equation (11) are:

$$
D\left(m_{1}, m_{2}\right)=0.7360, \quad D\left(m_{1}, m_{3}\right)=6.1923, \quad D\left(m_{2}, m_{3}\right)=0.6831
$$

Finally, the weights by Equation (14):

$$
\omega_{1}=0.4551, \quad \omega_{2}=0.0932, \quad \omega_{3}=0.4517
$$

According to the weights, a new set of data can be got by calculating:

$$
m\left(F_{1}\right)=0.06 \times 0.4551+0.02 \times 0.0932+0.02 \times 0.4517=0.0382
$$

In the same way, we can calculate $m\left(F_{2}\right)=0.5714, m\left(F_{3}\right)=0.1550, m\left(F_{4}\right)=0.0409, m(X)=$ 0.1944 .

Therefore, the decision can be made by fusing $\{0.0382,0.5714,0.1550,0.0409,0.1944\}$ with Dempster combination rule for 2 times, which are shown in Table 2. The results of other methods are also listed as a comparison. Following [25], we will use $\Delta=0.7$ as the threshold.

Since the fault $F_{2}$ has a belief degree of $89.18 \%$, it can be told that unbalance is the fault of the equipment. And compared with other methods, the new method performs much better. The belief entropy in Figure 2 is the smallest, which means that the proposed method has the smallest uncertain information volume. 
Table 2: Comparison of the result of several existing methods

\begin{tabular}{lcccccc}
\hline Combination rule & $F_{1}$ & $F_{2}$ & $F_{3}$ & $F_{4}$ & $\mathrm{X}$ & Diagnosis result \\
\hline Dempster [4,49] & 0.0205 & 0.5229 & 0.3933 & 0.0309 & 0.0324 & Uncertainty \\
Murphy [42] & 0.0112 & 0.6059 & 0.3508 & 0.0153 & 0.0168 & Uncertainty \\
Jiang [25] & 0.0111 & 0.7265 & 0.2313 & 0.0144 & 0.0168 & Unbalance \\
Deng [5] & 0.0111 & 0.7728 & 0.1851 & 0.0139 & 0.0165 & Unbalance \\
Proposed method & 0.0106 & 0.8918 & 0.0713 & 0.0115 & 0.0148 & Unbalance \\
\hline
\end{tabular}

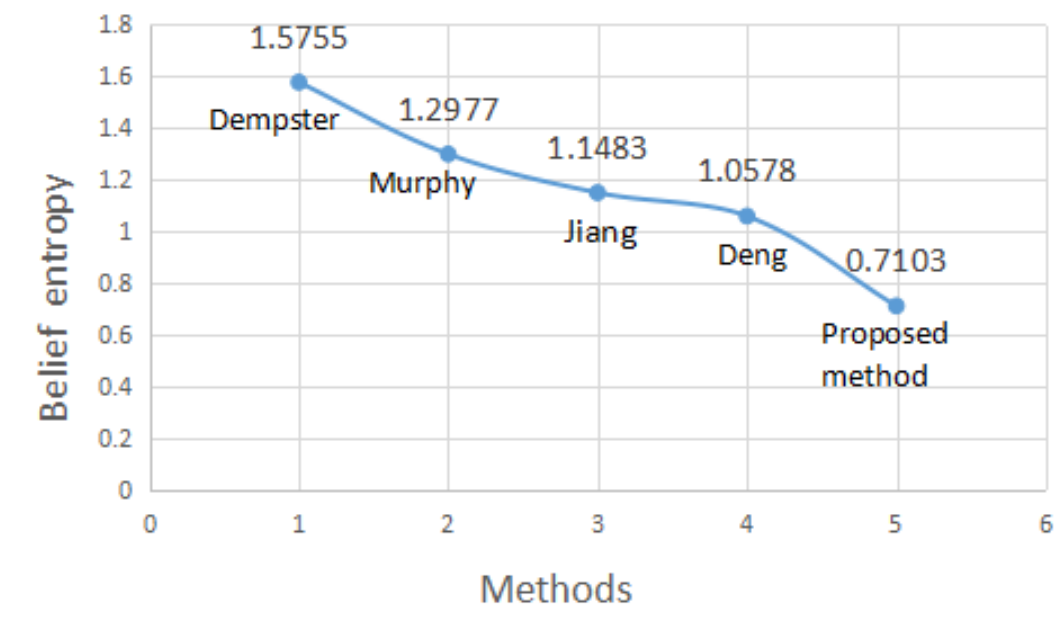

Figure 2: Belief entropy comparison.

\subsection{Example}

The proposed method is not only efficient in dealing with uncertainty, but also be falseevidence resilient. Suppose the system has collected five evidences from five different sensors [5], which are shown as follows:

$$
\begin{aligned}
& m_{1}: m_{1}(A)=0.5, m_{1}(B)=0.2, m_{1}(C)=0.3 ; \\
& m_{2}: m_{2}(A)=0, m_{2}(B)=0.9, m_{2}(C)=0.1 ; \\
& m_{3}: m_{3}(A)=0.55, m_{3}(B)=0.1, m_{3}(C)=0.35 ; \\
& m_{4}: m_{4}(A)=0.55, m_{4}(B)=0.1, m_{4}(C)=0.35 ; \\
& m_{5}: m_{5}(A)=0.55, m_{5}(B)=0.1, m_{5}(C)=0.35 ;
\end{aligned}
$$

The results are shown in Table 3, Obviously, the second evidence $m_{2}$ is conflicting with the others. Although there are more and more evidences that support $A$, Dempster's method cannot reach a reasonable result. $m(A)$ will always be zero as long as one evidence does not support it. While other methods revise the disadvantage. Apparently, the proposed method can have a probability 0.7663 to support $A$ when the third evidence is fused, and it always has the highest support.

In previous work, some information is not fully used. In our proposed method, not only the distance information between BPA, but also the difference of information volume of each $\mathrm{BPA}$ is considered. As a result, our method can efficiently measure the support degree of each sensor report. That is, if one report is more reliable, more weights will be assigned to this report. Therefore, the result of our improved method is more reasonable and more desirable.

Despite the advantages, the proposed method might not be very suitable in some situations. 
For example, the calculation process will be extremely complex if the data of evidence is huge. [25] considered belief entropy, and considered evidence distance, while our method considered both of them. Therefore, the calculation complexity might be twice as those methods. In addition, the proposed method might reach an unreasonable result if a bad evidence repeats many times. This is because the bad evidence cannot be identified, and they could have a mutual confirmation, leading to an illogical fusing result.

Table 3: Results of different method

\begin{tabular}{lcccc}
\hline Combination rule & $m_{1}, m_{2}$ & $m_{1}, m_{2}, m_{3}$ & $m_{1}, m_{2}, m_{3}, m_{4}$ & $m_{1}, m_{2}, m_{3}, m_{4}, m_{5}$ \\
\hline Dempster [4,49] & $\mathrm{m}(\mathrm{A})=0$ & $\mathrm{~m}(\mathrm{~A})=0$ & $\mathrm{~m}(\mathrm{~A})=0$ & $\mathrm{~m}(\mathrm{~A})=0$ \\
& $\mathrm{~m}(\mathrm{~B})=0.8571$ & $\mathrm{~m}(\mathrm{~B})=0.6316$ & $\mathrm{~m}(\mathrm{~B})=0.3288$ & $\mathrm{~m}(\mathrm{~B})=0.1228$ \\
Murphy [42] & $\mathrm{m}(\mathrm{C})=0.1429$ & $\mathrm{~m}(\mathrm{C})=0.3684$ & $\mathrm{~m}(\mathrm{C})=0.6712$ & $\mathrm{~m}(\mathrm{C})=0.8772$ \\
& $\mathrm{~m}(\mathrm{~A})=0.1543$ & $\mathrm{~m}(\mathrm{~A})=0.3500$ & $\mathrm{~m}(\mathrm{~A})=0.6027$ & $\mathrm{~m}(\mathrm{~A})=0.7958$ \\
& $\mathrm{~m}(\mathrm{~B})=0.7469$ & $\mathrm{~m}(\mathrm{~B})=0.5524$ & $\mathrm{~m}(\mathrm{~B})=0.2627$ & $\mathrm{~m}(\mathrm{~B})=0.0932$ \\
Jiang [25] & $\mathrm{m}(\mathrm{C})=0.0988$ & $\mathrm{~m}(\mathrm{C})=0.1276$ & $\mathrm{~m}(\mathrm{C})=0.1346$ & $\mathrm{~m}(\mathrm{C})=0.1110$ \\
& $\mathrm{~m}(\mathrm{~A})=0.4206$ & $\mathrm{~m}(\mathrm{~A})=0.6819$ & $\mathrm{~m}(\mathrm{~A})=0.8244$ & $\mathrm{~m}(\mathrm{~A})=0.8945$ \\
& $\mathrm{~m}(\mathrm{~B})=0.3944$ & $\mathrm{~m}(\mathrm{~B})=0.1310$ & $\mathrm{~m}(\mathrm{~B})=0.0326$ & $\mathrm{~m}(\mathrm{~B})=0.0071$ \\
Deng [5] & $\mathrm{m}(\mathrm{C})=0.1850$ & $\mathrm{~m}(\mathrm{C})=0.1871$ & $\mathrm{~m}(\mathrm{C})=0.1430$ & $\mathrm{~m}(\mathrm{C})=0.0984$ \\
& $\mathrm{~m}(\mathrm{~A})=0.1543$ & $\mathrm{~m}(\mathrm{~A})=0.5816$ & $\mathrm{~m}(\mathrm{~A})=0.8061$ & $\mathrm{~m}(\mathrm{~A})=0.8909$ \\
& $\mathrm{~m}(\mathrm{~B})=0.7469$ & $\mathrm{~m}(\mathrm{~B})=0.2439$ & $\mathrm{~m}(\mathrm{~B})=0.0481$ & $\mathrm{~m}(\mathrm{~B})=0.0086$ \\
& $\mathrm{~m}(\mathrm{C})=0.0988$ & $\mathrm{~m}(\mathrm{C})=0.1745$ & $\mathrm{~m}(\mathrm{C})=0.1457$ & $\mathrm{~m}(\mathrm{C})=0.1005$ \\
& $\mathrm{~m}(\mathrm{~A})=0.1543$ & $\mathrm{~m}(\mathrm{~A})=0.7663$ & $\mathrm{~m}(\mathrm{~A})=0.8554$ & $\mathrm{~m}(\mathrm{~A})=0.9063$ \\
& $\mathrm{~m}(\mathrm{~B})=0.7469$ & $\mathrm{~m}(\mathrm{~B})=0.0424$ & $\mathrm{~m}(\mathrm{~B})=0.0082$ & $\mathrm{~m}(\mathrm{~B})=0.0015$ \\
& $\mathrm{~m}(\mathrm{C})=0.0988$ & $\mathrm{~m}(\mathrm{C})=0.1913$ & $\mathrm{~m}(\mathrm{C})=0.1364$ & $\mathrm{~m}(\mathrm{C})=0.0922$ \\
\hline
\end{tabular}

\section{Conclusions}

In fault diagnosis and other sensor data fusion systems, the reports of different sensors may be influenced by some complex environments, leading them less reliable. Therefore, how to efficiently determine the reliability of each report, or to say, the weight of each report is very important. To address this issue, we propose an improved method based on the belief entropy and the evidence distance. The method considers both the degree of conflict and the difference of information volume among evidences. An application and an example illustrate the efficiency of the method in evidential sensor fusion. It shows that the proposed method is more efficient for highly conflicting evidences with better performance of convergence and less uncertainty, compared with the existing methods.

Some related advantages and disadvantages of different methods are discussed as follows.

- Dempster's method, which can well deal with imprecise and uncertain information, is widely used in fusing information. However, when it comes to highly conflicting evidences, the method will always lead to some illogical results.

- Murphy's method, which uses an average operation to combine the conflicting evidence, is able to deal with highly conflicting evidences to some extent. However, the difference and relationship of evidences is neglected.

- In Jiang's method, belief entropy is used to calculate the weight of each evidence. It considers the difference of evidence, which makes it more reasonable than Murphy's method. 
- In Deng's method, which is different from Jiang's method, evidence distance is used to calculate the weight rather than the belief entropy. And the similarity between two evidences is proposed.

- The proposed method, considering both belief entropy and evidence distance, has a better result. Although it might be not very suitable in some situations, it leverages the advantages of Jiang's method and Deng's method.

\section{Funding}

The work is partially supported by National Natural Science Foundation of China (Grant Nos. 61573290, 61503237).

\section{Author contributions. Conflict of interest}

The authors contributed equally to this work. The authors declare no conflict of interest.

\section{Bibliography}

[1] Abellan, J. (2017). Analyzing properties of Deng entropy in the theory of evidence, Chaos Solitons \& Fractals, 95, 195-199, 2017.

[2] An, J.Y.; Hu, M.; Fu, L.; Zhan, J.W. (2019). A novel fuzzy approach for combining uncertain conflict evidences in the Dempster-Shafer theory, IEEE Access, 7, 7481-7501, 2019.

[3] Cui, H.; Liu, Q.; Zhang, J.; Kang, B. (2019). An improved Deng entropy and its application in pattern recognition, IEEE Access, 7,18284-18292, 2019.

[4] Dempster, A.P. (1967). Upper and lower probabilities induced by a multivalued mapping, Annals of Mathematics and Statistics, 38(2), 325-339, 1967.

[5] Deng, Y.; Shi, W.K.; Zhu, Z.F.; Liu, Q. (2004). Combining belief functions based on distance of evidence, Decision Support Systems, 38(3), 489-493, 2004.

[6] Deng, Y. (2106). Deng entropy, Chaos, Solitons \& Fractals, 91, 549-553, 2016.

[7] Deng, W.; Deng, Y. (2018). Entropic methodology for entanglement measures, Physica A: Statistical Mechanics and its Applications, 512, 693-697, 2018.

[8] Deng, X.Y.; Deng, Y. (2019). D-AHP method with different credibility of information, Soft Computing, 23(2), 683-691, 2019.

[9] Deng, X.Y.; Jiang, W. (2019). D number theory based game-theoretic framework in adversarial decision making under a fuzzy environment, International Journal of Approximate Reasoning, 106, 194-213, 2019.

[10] Deng, X.Y.; Jiang, W.; Wang, Z. (2019). Zero-sum polymatrix games with link uncertainty: A Dempster-Shafer theory solution, Applied Mathematics and Computation, 340, 101-112, 2019.

[11] Dong, Y.K.; Wang, J.Y.; Chen, F.H.; Hu, Y.; Deng, Y. (2017). Location of Facility Based on Simulated Annealing and "ZKW" Algorithms, Mathematical Problems in Engineering, 2017, 9, 2017. 
[12] Dubois, D.; Prade, H. (1988). Representation and combination of uncertainty with belief functions and possibility measures, Computational Intelligence, 4(3), 244-264, 1988.

[13] Dutta, P. (2017). Modeling of variability and uncertainty in human health risk assessment, MethodsX, 4, 76-85, 2017.

[14] Dutta, P. (2018). An uncertainty measure and fusion rule for conflict evidences of big data via Dempster-Shafer theory, International Journal of Image and Data Fusion, 9(2), 152-169, 2018.

[15] Dzitac, I.; Filip, F.G.; Manolescu, M.J. (2017). Fuzzy logic is not fuzzy: World-renowned computer scientist Lotfi A. Zadeh, International Journal of Computers Communications \& Control, 12(6), 748-789, 2017.

[16] Fan, X.F.; Zuo, M.J. (2006). Fault diagnosis of machines based on D-S evidence theory. Part 1: D-S evidence theory and its improvement, Pattern Recognition Letters, 27(5), 366-376, 2006.

[17] Fei, L.G.; Deng, Y. (2019). A new divergence measure for basic probability assignment and its applications in extremely uncertain environments, International Journal of Intelligent Systems, 34(4), 584-600, 2019.

[18] Fei, L.; Deng, Y. (2018). Identifying influential nodes in complex networks based on the inverse-square law, Physica A: Statistical Mechanics and its Applications, 512, 1044-1059, 2018 .

[19] Gao, X.; Deng, Y. (2019). The negation of basic probability assignment, IEEE Access, 7, 10.1109/ACCESS.2019.2901932, 2019.

[20] Haenni, R. (2002). Are alternatives to Dempster's rule of combination real alternatives?: Comments on: About the belief function combination and the conflict management problem-Lefevre et al, Information Fusion, 3(3), 237-239, 2002.

[21] Han, Y.; Deng, Y. (2019). A novel matrix game with payoffs of Maxitive Belief Structure, International Journal of Intelligent Systems, 34(4), 690-706, 2019.

[22] Han, Y.; Deng, Y. (2018). A hybrid intelligent model for Assessment of critical success factors in high risk emergency system, Journal of Ambient Intelligence and Humanized Computing, 9(6), 1933-1953, 2018.

[23] Han, Y.; Deng, Y. (2018). An Evidential Fractal AHP target recognition method, Defence Science Journal, 68(4), 367-373, 2018.

[24] Jaunzemis, A.D.; Holzinger, M.J.; Chan, M.W.; Shenoy, P.P. (2019). Evidence gathering for hypothesis resolution using judicial evidential reasoning, Information Fusion, 49, 26-45, 2019.

[25] Jiang, W.; Wei, B.Y.; Xie, C.H.; Zhou, D.Y. (2016). An evidential sensor fusion method in fault diagnosis, Advances in Mechanical Engineering, 8(3), 1-7, 2016.

[26] Jiang, W. (2018). A correlation coefficient for belief functions, International Journal of Approximate Reasoning, 103, 94-106, 2018.

[27] Jiang, W.; Wang, S.Y. (2017). An Uncertainty Measure for Interval-valued Evidences, International Journal of Computers Communications \& Control, 12(5), 631-644, 2017. 
[28] Jin, L.Q.; Fang, X. (2017). Interval Certitude Rule Base Inference Method using the Evidential Reasoning, International Journal of Computers Communications \& Control, 12(6), 2017.

[29] Jousselme, A.L.; Grenier, D.; Bossé, É, (2001). A new distance between two bodies of evidence, Information Fusion, 2(2), 91-101, 2001.

[30] Kang, B.Y.; Zhang, P.D.; Gao, Z.Y.; Chhipi-Shrestha, G.; Hewage, K.; Sadiq, R. (2019). Environmental assessment under uncertainty using Dempster-Shafer theory and Z-numbers, Journal of Ambient Intelligence and Humanized Computing, https://doi.org/10.1007/s12652-019-01228-y, 2019.

[31] Kang, B.Y.; Deng, Y.; Hewage, K.; Sadiq, R. (2019). A method of measuring uncertainty for Z-number, IEEE Transactions on Fuzzy Systems, 27(4), 731-738, 2019.

[32] Kuzemsky, A.L. (2018). Temporal evolution, directionality of time and irreversibility, Rivista Del Nuovo Cimento, 41(10), 513-574, 2018.

[33] Lefevre, E.; Colot, O.; Vannoorenberghe, P. (2002). Belief function combination and conflict management, Information fusion, 3(2), 149-162, 2002.

[34] Li, M.Z.; Zhang, Q.; Deng, Y. (2018). Evidential identification of influential nodes in network of networks, Chaos, Solitons \& Fractals, 117, 283-296, 2018.

[35] Li, Y.X.; Deng, Y. (2018). Generalized Ordered Propositions Fusion Based on Belief Entropy, International Journal of Computers Communications \& Control, 13(5), 792-807, 2018.

[36] Liu, C.;Li, L.; Wang, Z.; Wang, R.(2019). Pattern transitions in a vegetation system with cross-diffusion, Applied Mathematics and Computation, 342, 255-262, 2019.

[37] Liu, H.; Dzitac, I.; Guo, S.(2018). Reduction of Conditional Factors in Causal Analysis, International Journal of Computers Communications \& Control, 13(3), 383-390, 2018.

[38] Meng, D.; Yang, S.; Zhang, Y.; Zhu, S (2018). Structural reliability analysis and uncertainties-based collaborative design and optimization of turbine blades using surrogate model, Fatigue ES Fracture of Engineering Materials \& Structures, 10.1111/ffe.12906, 2018.

[39] Meng, D.; Liu, M.; Yang, S.; Zhang, H.; Ding, R. (2018). A fluid-structure analysis approach and its application in the uncertainty-based multidisciplinary design and optimization for blades, Advances in Mechanical Engineering, 10(6), 1687814018783410, 2018.

[40] Meng, D.; Li, Y.; Zhu, S.; Lv, G.; Correia, J.; de Jesus, A.(2019). An Enhanced Reliability Index Method and Its Application in Reliability-Based Collaborative Design and Optimization, Mathematical Problems in Engineering, https://doi.org/10.1155/2019/4536906, 2019.

[41] Mo, H.M.; Deng, Y. (2019). An evaluation for sustainable mobility extended by D numbers, Technological and Economic Development of Economy, Accepted, 2019.

[42] Murphy, C.K. (2000); Combining belief functions when evidence conflicts, Decision Support Systems, 29(1), 1-9, 2000.

[43] Pan, L.P.; Deng, Y. (2018). A New Belief Entropy to Measure Uncertainty of Basic Probability Assignments Based on Belief Function and Plausibility Function, Entropy, 20(11), $842,2018$. 
[44] Paté-Cornell, M.E. (1990). Organizational Aspects of Engineering System, Safety Science, 250, 1210-16, 1990.

[45] Rong, H.; Ge, M.; Zhang, G.; Zhu, M. (2018). An approach for detecting fault lines in a small current grounding system using fuzzy reasoning spiking neural p systems, International Journal of Computers Communications \& Control, 13(4), 521-536, 2018.

[46] Sabahi, F. (2016). A Novel Generalized Belief Structure Comprising Unprecisiated Uncertainty Applied to Aphasia Diagnosis, Journal of Biomedical Informatics, 62, 66-77, 2016.

[47] Seiti, H.; Hafezalkotob, A. (2019). Developing the R-TOPSIS methodology for risk-based preventive maintenance planning: A case study in rolling mill company, Computers \& Industrial Engineering, 128, 622-636, 2019.

[48] Seiti, H.; Hafezalkotob, A.; Martinez, L. (2019). R-numbers, a new risk modeling associated with fuzzy numbers and its application to decision making, Information Sciences, 483, 206$231,2019$.

[49] Shafer, G. (1967). A Mathematical Theory of Evidence, Princeton University Press, 1967.

[50] Smets, P. (1993). Belief functions: the disjunctive rule of combination and the generalized Bayesian theorem, International Journal of Approximate Reasoning, 9(1), 1-35, 1993.

[51] Smets, P.; Kennes, R. (1994). The transferable belief model, Artificial Intelligence, 66(2), 191-234, 1994.

[52] Song, Y.T.; Deng, Y. (2019). A new method to measure the divergence in evidential sensor data fusion, International Journal of Distributed Sensor Networks, 15(4), DOI: 10.1177/1550147719841295, 2019.

[53] Su, X.Y.; Li, L.S.; Shi, F.J.; Qian, H. (2018). Research on the Fusion of Dependent Evidence Based on Mutual Information, IEEE Access, 6, 71839-71845, 2018.

[54] Su, X.Y.; Li, L.S.; Qian, H.; Sankaran, M.; Deng, Y. (2019). A new rule to combine dependent bodies of evidence, Soft Computing, https://doi.org/10.1007/s00500-019-03804-y, 2019.

[55] Sun, R.L.; Deng, Y. (2019); A new method to identify incomplete frame of discernment in evidence theory, IEEE Access, 7(1), 15547-15555, 2019.

[56] Sun, R.L.; Deng, Y. (2019). A new method to determine generalized basic probability assignment in the open world, IEEE Access, 7(1), accepted, 2019.

[57] Vandoni, J.; Aldea, E.; Le Hégarat-Mascle, S. (2019). Evidential query-by-committee active learning for pedestrian detection in high-density crowds, International Journal of Approximate Reasoning, 104, 166-184, 2019.

[58] Wang, T.; Zhang, G.X.; Rong, H.N.; Pérez-Jiménez, M.J. (2014). Application of fuzzy reasoning spiking neural $\mathrm{P}$ systems to fault diagnosis, International Journal of Computers Communications \& Control, 9(6), 786-799, 2014.

[59] Wang, Y.; Wang, S.; Deng, Y. (2019). A modified efficiency centrality to identify influential nodes in weighted networks, Pramana, 68(4), 68, 2019. 
[60] Wang, Y.J.; Deng, Y. (2018). Base belief function: an efficient method of conflict management, Journal of Ambient Intelligence and Humanized Computing, https://doi.org/10.1007/s12652-018-1099-2, 2018.

[61] Wang, J.; Qiao, K.Y.; Zhang, Z.Y. (2019). An improvement for combination rule in evidence theory, Future Generation Computer Systems, 91, 1-9, 2019.

[62] Wei, B.; Deng, Y. (2018). A cluster-growing dimension of complex networks: From the view of node closeness centrality, Physica A: Statistical Mechanics and its Applications, 10.1016/j.physa.2019.01.125, 2019.

[63] Xiao, F.Y. (2018). A Hybrid Fuzzy Soft Sets Decision Making Method in Medical Diagnosis, IEEE Access, 6, 25300-25312, 2018.

[64] Xiao, F.Y. (2018); A novel multi-criteria decision making method for assessing health-care waste treatment technologies based on D numbers, Engineering Applications of Artificial Intelligence, 71(2018), 216-225, 2018.

[65] Xiao, F.Y. (2019). A multiple criteria decision-making method based on D numbers and belief entropy, International Journal of Fuzzy Systems, https://doi.org/10.1007/s40815-01900620-2, 2019.

[66] Xiao, F.Y.; Ding, W.P. (2019). A novel multi-criteria decision making method for assessing health-care waste treatment technologies based on D numbers, Applied Soft Computing, 74, DOI: 10.1016/j.asoc.2019.03.043, 2019.

[67] Xu, X.B.; Li, S.B.; Song, X.J.; Wen, C.L.; Xu, D.L. (2016). The optimal design of industrial alarm systems based on evidence theory, Control Engineering Practice, 46, 142-156, 2016.

[68] Xu, H.; Deng, Y. (2019). Dependent Evidence Combination Based on DEMATEL Method, International Journal of Intelligent Systems, 34, 10.1002/int.22107, 2019.

[69] Xu, P.; Zhang, R.; Deng, Y. (2019). A Novel Visibility Graph Transformation of Time Series into Weighted Networks, Chaos, Solitons \& Fractals, 2018, 201-208, 2018.

[70] Yager, R.R. (1987). On the Dempster-Shafer framework and new combination rules, Information Sciences, 41(2), 93-137, 1987.

[71] Yang, H.C.; Deng, Y.; Jones, J. (2018). Network Division Method Based on Cellular Growth and Physarum-inspired Network Adaptation, International Journal of Unconventional Computing, 13(6), 477-491, 2018.

[72] Yin, L.K.; Deng, Y. (2018). Toward uncertainty of weighted networks: An entropy-based model, Physica A: Statistical Mechanics and its Applications, 508, 176-186, 2018.

[73] Yin, L.K.; Deng, X.Y.; Deng, Y. (2019). The negation of a basic probability assignment, IEEE Transactions on Fuzzy Systems, 27(1), 135-143, 2019.

[74] Zadeh, L.A. (1965). Fuzzy Sets, Information and Control, 8(3), 338-353, 1965.

[75] Zadeh, L.A. (1986). A simple view of the Dempster-Shafer theory of evidence and its implication for the rule of combination, AI magazine, 7(2), 85, 1986.

[76] Zavadskas, E.K.; Antucheviciene, J.; Hajiagha, S.H.R. (2015). The interval-valued intuitionistic fuzzy MULTIMOORA method for group decision making in engineering, Mathematical Problems in Engineering, 2015, 13, 2015. 
[77] Zavadskas, E.K.; Antucheviciene, J.; Turskis, Z.; Adeli, H. (2016). Hybrid multiple-criteria decision-making methods: A review of applications in engineering, Scientia Iranica. Transaction A, Civil Engineering, 23(1), 1, 2016.

[78] Zhang, L.M.; Wu, X.G.; Qin, Y.W.; Skibniewski, M.J.; Liu, W.L. (2016). Towards a Fuzzy Bayesian Network Based Approach for Safety Risk Analysis of Tunnel-Induced Pipeline Damage, Risk Analysis, 36(2), 278-301, 2016.

[79] Zhang, L.M.; Wu, X.G.; Zhu, H.P.; AbouRizk, S.M. (2017). Perceiving safety risk of buildings adjacent to tunneling excavation: An information fusion approach, Automation in Construction, 73, 88-101, 2017.

[80] Zhang, W.Q.; Deng, Y. (2018). Combining conflicting evidence using the DEMATEL method, Soft computing, https://doi.org/10.1007/s00500-018-3455-8, 2018.

[81] Zhang, H.P.; Deng, Y. (2018). Engine fault diagnosis based on sensor data fusion considering information quality and evidence theory, Advances in Mechanical Engineering, 10(11), DOI: 10.1177/1687814018809184, 2018.

[82] Zhao, D.; Wang, L.; Wang, Z.; Xiao, G. (2019). Virus Propagation and Patch Distribution in Multiplex Networks: Modeling, Analysis, and Optimal Allocation, IEEE Transactions on Information Forensics and Security, 14(7), 1755-1767, 2019.

[83] Zhu, W.B.; Yang, H.C.; Jin, Y.; Liu, B.Y. (2017). A Method for Recognizing Fatigue Driving Based on Dempster-Shafer Theory and Fuzzy Neural Network, Mathematical Problems in Engineering, 10, 2017. 